Introduction With increasing levels of psychological distress in adolescents, it is even more important that teachers know how to identify, support and refer young people facing mental health difficulties. The catchment area of our hospital includes a significant population of young people attending Jewish faith schools. We describe how our service developed an inclusive, social and hands-on approach to improving the confidence and knowledge of schools within this population addressing issues surrounding mental health.

Method Teachers from nine Jewish schools located within the hospital's catchment area were invited to attend a Mental Health Seminar, delivered by a multidisciplinary team of psychiatrists, psychologists and teachers from the Hospital School experienced in matters of mental health. Participants were allocated to tables by discipline and type of school to ensure distribution of expertise. Six vignettes addressing a range of common mental health scenarios were distributed amongst the group. Each table was then assigned a specific vignette and tasked to identify the issue raised and to suggest a course of action. Each table then took turns to feedback back their work for group discussion.

Results $100 \%$ of participants agreed knowledge and understanding of issues around mental health were improved.

Conclusions We gained good insight into the needs of the community to plan for future meetings. The seminar was deemed wholly worthwhile and a useful way to discuss attitudes towards mental health between orthodox and non-orthodox schools within the community. The seminar helped improve both identifying the signs of a number of mental health conditions and how to make referrals to specialist services.

\section{G259(P) MENTAL HEALTH MATTERS: IMPROVING THE CARE OF OUR PAEDIATRIC PATIENTS}

P de Sousa, N Lord, M Digby, E Parker, AM Buckley, L Adenuga. Paediatrics, East Surrey Hospital, Redhill, UK

\subsection{6/archdischild-2020-rcpch.223}

Aims Affective disorders in young people are rising described as an 'Adolescent Mental Health Crisis' ${ }^{1}$ with a $68 \%$ increase in hospital self-harm presentations in 13-16 year old girls between 2011 and 2014. ${ }^{1}$ Paediatric doctors and nurses at East Surrey Hospital felt the numbers were increasing locally and that they were inadequately trained to manage this group of patients. To better understand the needs of young people and improve the care we delivered, the 'Mental Health Working Group' was set up locally in 2018. This was made up of paediatric doctors, mental health liaison nurses and ward managers. Our objectives were multi-fold but one of the early tasks was to: 1) Audit patients admitted with mental health problem, 2) Design + Introduce a Proforma/Risk Assessment 3) Re-audit.

Methods Initial audit period from February to April 2018 and included all patients presenting with mental health problems. The Clerking Proforma and Risk Assessment were introduced in October 2018 to guide doctors in their documentation and management of mental health patients:

- All patients presenting with a mental health problem must be clerked using the proforma + risk assessment form.

- Risk Assessment must be complete once every 12 hours or more if a patient's mental state changes.
- Feedback on the proforma was obtained from all staff members before its introduction and from a Young Senior Participation Officer from the local council.

The re-audit was from November to January 2019 and included subjective feedback from doctors.

Results

- Doctors rated the introduction of the proforma and risk assessment 4.3/5.

- $76 \%$ of the Proformas were completed on admission

- $74 \%$ had a Risk Assessment form completed on admission

- $78 \%$ of the clerking proforma was competed correctly

- $11 \%$ had a risk assessment completed by a Doctor on ward round

- $68 \%$ had a risk assessment completed by a Nurse

\begin{tabular}{lll}
\hline & Feb-April 2018 & Nov-Jan 2019 \\
\hline Number of Admissions & 41 & 24 \\
Suicidal ideation Documented? & $40 \%$ & $100 \%$ \\
Capacity Documented & $0 \%$ & $94 \%$ \\
Risk Assessment Documented & $10 \%$ & $74 \%$ \\
\hline
\end{tabular}

Conclusion The proforma has led to improved documentation and better risk assessments. The Mental Health Working Group has overall reduced the stigma amongst staff members, helped to increase their confidence in managing young inpatients and highlighted the 'national crisis' and need for more funding.

\section{G260(P) MENTAL HEALTH SIMULATION FOR PAEDIATRIC JUNIOR DOCTORS: USING LOW TECHNOLOGY, HIGH FIDELITY SIMULATION TO ENHANCE CLINICIAN CONFIDENCE IN PAEDIATRIC MENTAL HEALTH CARE}

O Sanwo, M Dubus. Paediatrics, East Kent Hospitals University NHS FT, Kent, UK

\subsection{6/archdischild-2020-rcpch.224}

Background In the 2017 Mental Health of Children and Young People (CYP) in England Report, 15.4\% of children presented to a physical health specialist, such as a paediatrician, regarding their mental health. An Australian study found that over a 7-year period, childhood mental health presentations to Emergency Departments increased three times faster than physical health presentations, (Hiscock et al, 2018). However, trainee paediatricians receive little formal training on the assessment and management of common mental health presentations. Despite the clinical and educational advantages of simulation, its use is less common in mental health (Attoe et al, 2016). Consequently, little is known of the feasibility and effect of a paediatric mental health simulation programme for junior doctors working in paediatrics.

Aim To ascertain whether the introduction of a paediatric mental health simulation programme would improve junior doctor confidence in the assessment and management of common acute paediatric mental health presentations.

Method Two simulation scenarios on self-harm and anorexia nervosa were developed and incorporated into the weekly insitu simulation programme for junior doctors in paediatrics trust-wide over 6 months. The debrief after each simulation 
included a discussion on risk assessment and management, signposting to appropriate resources and sharing learning points. Confidence in history-taking, psychosocial assessment and management were assessed by self-report before and after the simulation.

Results Following both the self-harm and anorexia nervosa scenarios respectively, there were increases in clinician-reported confidence in history taking $(37 \%$ vs. $85.2 \% ; 41.7 \%$ vs. $70 \%)$, psychosocial assessment $(34.6 \%$ vs. $67.8 \%)$ and management (37\% vs. $60.7 \% ; 58.3 \%$ vs. $63.7 \%)$. Thematic analysis of qualitative feedback highlighted the desire for further training as well as various benefits of simulation.

Conclusion As the mental health needs of CYP continue to rise, it is clear that there remains an urgent and unmet need for mental health training for paediatric clinicians. This data suggests that low technology high fidelity simulation is a feasible method of improving clinician confidence in the assessment and management of CYP with mental health presentations. It is hoped that by developing such programmes, clinicians will be helped to cultivate the necessary skills to appropriately assess and manage patients presenting with mental health concerns.

\section{G261(P) HOW CONFIDENT DO PAEDIATRICIANS FEEL IN MANAGING MEDICALLY UNEXPLAINED SYMPTOMS?}

J Hoddes, I Takon. Paediatrics, East and North Hertfordshire NHS Trust, Stevenage, UK

\subsection{6/archdischild-2020-rcpch.225}

Aims This survey was designed to explore the experience and confidence of Paediatricians in the management of children and young people with Medically Unexplained Symptoms (MUS).

Methods Semi- structured questionnaires using mostly closed ended questions regarding clinicians' experience of managing cases of MUS and their confidence in managing such cases were distributed to all middle grade doctors and consultants working in acute and community paediatrics within our trust Results 17 completed questionnaires were analysed: 6 Middle Grade Doctors (MGD); 7 Hospital Consultants (HC) and 4 Community Consultants (CC). Data was subcategorised and frequencies of responses determined.

5/17 respondents, all hospital based, reported seeing children with MUS on a monthly basis.

$5 / 17$ respondents were confident in making a diagnosis of MUS (4 hospital and 1 community consultant). All Middle Grade Doctors did not feel confident in diagnosing MUS and all but one Middle Grade respondent felt uncomfortable discussing MUS with patients and families.

9/17 ( 53\%) of respondents strongly agreed with having an end point for investigations with 5 out of 9 respondents being hospital consultants.

Hospital Consultants and Middle Grade Doctors tended to believe that it was their role to rule out a physical cause before addressing mental health issues however Community Consultants were less likely to agree with this view.

The general trend amongst Paediatricians was towards sharing uncertainties about the diagnosis with the family but there was a diverse range of opinion on this irrespective of workplace or level of experience. All groups would raise the possibility of MUS with colleagues earlier than they would with the families.
Consultants (HC and CC) were generally likely to pre-empt normal test results with the family.

Community Consultants were the group most likely to feel that they had already received some training in MUS, however, several CC confused the diagnosis of MUS with Factitious Induced Illness in their comments.

Conclusions MUS causes high levels of morbidity within the paediatric population; however, our survey has shown that many Paediatricians of all levels do not feel confident in managing MUS. We advocate that training in Medically Unexplained Symptoms is mandatory for all Paediatricians.

\section{G262(P) MENTAL HEALTH MATTERS: THE IMPACT OF INCLUDING MENTAL HEALTH PROFESSIONALS WITHIN A GP-BASED CHILD HEALTH MULTIDISCIPLINARY TEAM}

I Talintyre, S Adnan, K Aitchison. Integrated Paediatrics, St Mary's Hospital, London, UK

\subsection{6/archdischild-2020-rcpch.226}

Aim The majority of mental health problems start in childhood and adolescence, but 3 in 4 children with a diagnosable mental health condition do not get access to the support they need. ${ }^{1}$ In order to address this deficit, we aimed to evaluate the impact of including mental health (CAMHS) professionals within a GP-based child health multidisciplinary team (MDT). A component of an existing child health GP hub model, these monthly MDT meetings are opportunities for GPs, specialists and allied healthcare professionals to discuss paediatric cases in primary care, providing integrated care with previously demonstrated increased connections and shared learning between professionals. $^{2}$

Methods 2 questionnaires were developed for GPs at 2 hub MDTs and distributed just prior to and 9 months after the introduction of a CAMHS professional. The attending CAMHS professional gave written feedback on their experience.

Results 14 questionnaires were returned by GPs. Since the inclusion of a CAMHS professional within the MDT, 100\% agreed/strongly agreed they added value to case discussions and felt comfortable contacting them for advice outside the MDT, and 75\% agreed their own awareness of services and resources available had increased.

Both pre- and post-intervention, 50\% of GPs disagreed/ strongly disagreed that they were confident managing paediatric patients with mental health problems on their own. The most commonly reported barriers to managing these patients in the community related to access (60\%) and waiting time (40\%) for secondary care.

The main themes in the CAMHS professional's reflections of attending the MDT were collaboration, building relationships with referrers, and mutual learning.

Conclusion The inclusion of a CAMHS professional in the child health hub MDT improved relationship-building and knowledge-sharing between professionals. However, GPs continue to lack confidence managing paediatric mental health. Further innovation is required to tackle barriers to accessing support including waiting time and limited resources.

\section{REFERENCES}

1. YoungMinds. YoungMinds Impact Report 2018-19. Available from: https://youngminds.org.uk/about-us/reports/our-impact-reports/

2. Montgomery-Taylor S, Watson M, Klaber R. Child Health General Practice Hubs: service evaluation. Archives of Disease in Childhood. 2016;101:333-337. 